\title{
Accommodation of vascularized xenografts: Expression of "protective genes" by donor endothelial cells in a host Th2 cytokine environment
}

\author{
Fritz H. Bach ${ }^{1}$, Christiane Ferran ${ }^{1}$, Paul HeChenleitner ${ }^{1}$, Walter Mark ${ }^{1}$, \\ Nozomi Koyamada, Tsukasa Miyatake, Hans WinkLeR ${ }^{1}$, AnNe Badrichani ${ }^{1}$, \\ DANIEL CANDINAS ${ }^{1} \&$ WAYNE W. HANCOCK ${ }^{1,2}$

\begin{abstract}
${ }^{1}$ Sandoz Center for Immunobiology, Department of Surgery, and ${ }^{2}$ Department of Pathology, New England Deaconess Hospital and Harvard Medical School, 99 Brookline Avenue, Boston, Massachusetts 02215, USA
\end{abstract} \\ Correspondence should be addressed to F.H.B.
}

\begin{abstract}
Organ xenografts under certain circumstances survive in the presence of anti-graft antibodies and complement, a situation referred to as "accommodation." We find that the endothelial cells (ECs) in hamster hearts that accommodate themselves in rats express genes, such as $A 20$ and $b c l-$ 2, that in vitro protect ECs from apoptosis and prevent upregulation in those cells of proinflammatory genes such as cytokines, procoagulant and adhesion molecules. Hearts that are rejected do not express these genes. In addition, vessels of rejected hearts show florid transplant arteriosclerosis whereas those of accommodated hearts do not. Accommodated xenografts have an ongoing T helper cell type 2 (Th2) cytokine immune response, whereas the rejected grafts have a Th1 response. We propose a model for factors that contribute to the survival of xenografts and the avoidance of transplant arteriosclerosis.
\end{abstract}

An immediately revascularized organ transplant, such as a heart or kidney, is usually rejected when the recipient has complementfixing anti-graft antibodies, as can occur with an ABO-incompatible allograft. However, several investigators have demonstrated that lowering the titer of the offending anti-ABO antibodies before transplantation, and maintaining such lowered levels for several days post engraftment, allowed allograft survival even when the antibodies were later permitted to return to predepletion levels and in the presence of normal levels of complement ${ }^{1,2}$. We proposed use of the term "accommodation" for the survival of a vascularized transplant despite the presence of anti-graft antibodies and complement. This definition allows for the possible involvement of both host and graft factors in determining survival. As such, one possible mechanism to explain accommodation, and one consistent with the definition of accommodation, is that the isotype of the antibodies present in a host carrying an accommodated organ and binding to the endothelium of that organ are antibodies that would not lead to rejection under any circumstances. We suggested that under certain circumstances it may be possible to achieve accommodation of xenografts ${ }^{3,4}$.

In the case of a xenograft, the recipient either has preexisting anti-donor antibodies that together with complement cause hyperacute rejection (discordant combinations such as pig to primate) or generates anti-graft antibodies over the several days after transplantation that lead to rejection (concordant transplants such as hamster to rat, or baboon to human). In all instances when the donor organ survives for three or more days, endothelial cell (EC) activation is noted before organ rejection. Signs of EC activation include the upregulation of multiple proinflammatory genes, including those that encode adhesion molecules, cytokines, tissue factor and others ${ }^{5,6}$.

We have studied a hamster-to-rat cardiac transplant model in which the recipient is treated for 6 to 11 days with cobra venom factor (CVF) to block complement, and daily with cyclosporin A (CyA); this protocol results in accommodation of approximately $75 \%$ of grafts. There are three findings of note by immunopathologic analysis. First, there is strong expression in ECs and smooth muscle cells (SMCs) of the accommodated organs of four genes, hemoxygenase, $A 20, b c l-2$ and $b c l-x_{1}$ that fit the definition of "protective genes": that is, genes that when expressed block the activation of nuclear factor kappa B (NF-kB) and in some cases prevent apoptosis. These protective genes are not present in ECs or SMCs of the rejected hearts, which, however, express the Bad, Bax and CPP-32 gene products that counter the effects of antiapoptotic genes and are thus pro-apoptotic. Second, the accommodated hearts contain a dense mononuclear cell infiltrate and a Th 2 cytokine response, whereas the rejected hearts have a Th1 response. Last, high titers of IgG2c (an IgG that fixes complement poorly) are deposited on the ECs of accommodated grafts, but are essentially not detected within rejected xenografts. Coincident with these findings, we find that the grafts that are rejected show evidence of transplant arteriosclerosis (chronic rejection), whereas the accommodated hearts do not.

We hypothesize that the development of accommodation and avoidance of transplant arteriosclerosis in this model is related to the effects mediated by these three factors: protective genes in the ECs of the graft, Th2 cytokines and IgG2c. The findings provide a possible molecular basis for accommodation; to the extent that these findings are relevant to long-term graft survival and the prevention of transplant arteriosclerosis, new approaches to overcoming xenograft rejection can be attempted.

In vitro studies of protective genes

A novel finding in this study relates to the in vivo expression in 

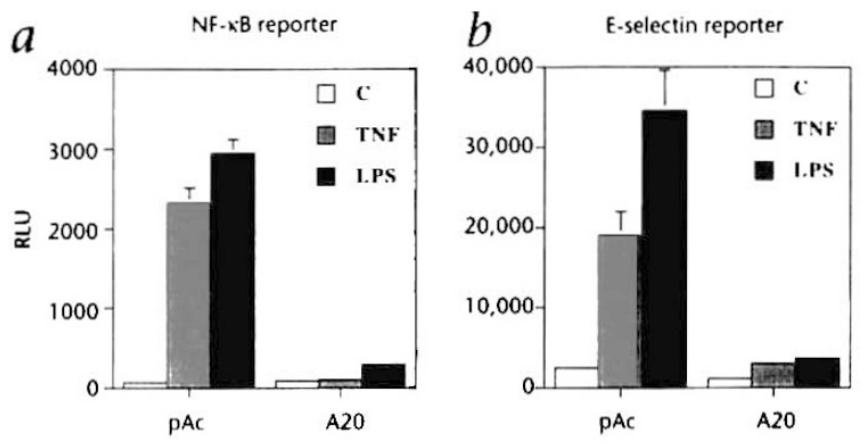

C
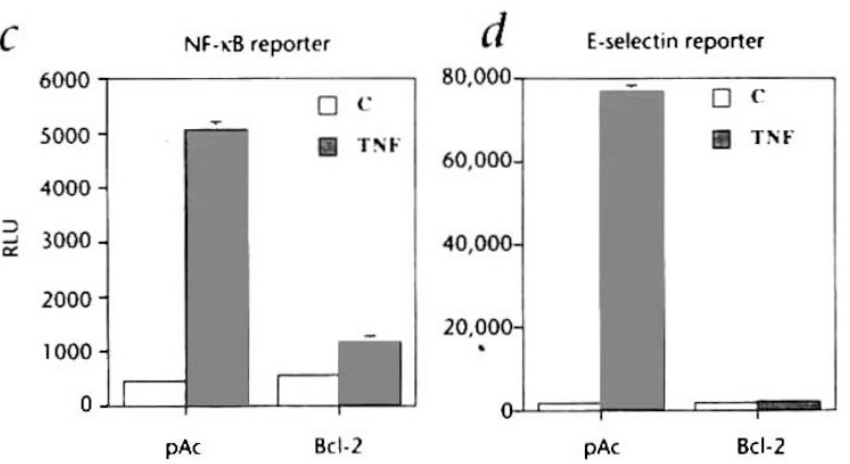

ECs of the accommodated organs of protective genes; that is, genes that prevent the upregulation of proinflammatory genes and certain genes that prevent the cells from undergoing apoptosis. We have studied three of these genes, $A 20, b c l-2$ and $b c l-x_{\mathrm{L}}$, which were all initially described on the basis of their anti-apoptotic properties ${ }^{7-9}$. Expression of $\mathrm{A2O}$ or $\mathrm{bcl}-2$ in ECs stimulated with tumor necrosis factor (TNF) in vitro inhibits the activation of a reporter that is dependent solely on $\mathrm{NF}-\mathrm{\kappa B}$ for its activation (Fig. 1, $a$ and $c$, respectively). Consistent with this finding, expression of $A 20$ or $b c l-2$ blocks the induction of an E-selectin reporter (Fig. 1, $b$ and $d$, respectively), a result representative of studies with reporters for interleukin-8 (IL-8), IкB $\alpha$ and tissue factor as well ${ }^{10-12}$. The inhibition of NF- $\mathrm{kB}$ and of the proinflammatory genes, such as E-selectin, are in all likelihood related in that the upregulation of E-selectin, as well as a number of other proinflammatory genes that are induced with EC activation, is strongly NF- $\mathrm{KB}$-dependent ${ }^{13-16}$.

Expression of $b c l-x_{1}$, which belongs to the same gene family as $b c l-2$, has the same consequences when expressed in ECs as expression of $b c l-2$ or $A 20$ on the response of the NF- $\kappa B$ reporter and reporters for the proinflammatory genes listed above (data not shown). $A 20, b c l-2$ and $b c l-x_{1}$, thus, all fulfill the criteria of a protective gene: ECs are protected from apoptosis and blocked from upregulating multiple inflammation-causing genes.

\section{Cardiac xenograft model}

Hamster hearts transplanted into unmodified rat recipients were rejected in an average of 3 days, as were xenografts transplanted into rats that were not splenectomized and that were treated with CyA ( $15 \mathrm{mg} / \mathrm{kg}$ body weight per day) to block host T-cell responses. Xenografts transplanted into rats treated with CVF only (60 units/ $\mathrm{kg}$ on day -1 and 20 units/ $\mathrm{kg}$ per day thereafter) to deplete complement survived for an average of 4.5 days.

In our first protocol, we treated rats for $\mathbf{1 1}$ days (from day $\mathbf{- 1}$ to day 9) with CVF and continuously with CyA. Two groups were studied to determine whether splenectomy affected the develop-
Fig. $1 a$, Inhibition of NF-KB by A20: $5 \times 10^{5}$ bovine aortic endothelial cells (BAECs) were cotransfected by the lipofectamine method with the NF$\kappa B$-luciferase reporter construct $(0.7 \mu \mathrm{g})$ as well as either the $A 20$ expression plasmid or the pAC control plasmid $(0.5 \mu \mathrm{g})$. The RSV $\beta$-galactosidase plasmid $(0.3 \mu \mathrm{g})$ was used in addition to correct for transfection efficiency. A20 expression abrogated the 12 - and 28 -fold induction of reporter activity in response to TNF and LPS, respectively. C refers to control (untreated) cells. RLU refers to relative light units and corresponds to luciferase values corrected by $\beta$-galactosidase values. See the Methods section for further experimental details. $\quad b$, Inhibition of E-selectin induction: as for part $a$, except that the E-selectin-luciferase reporter construct was used instead of the NF- $\kappa B$ construct. In the pAc control, induction with either TNF or LPS led to an 8- and 14-fold increase in the activity of the E-selectin reporter, respectively. A20 expression totally inhibited the inducibility of this reporter to background ( $\mathrm{pAc}$ ) levels. c, Cotransfection of $b c \mathrm{l}-2$ together with the NF$K B$ reporter to a very great extent abrogated the 10 -fold induction of this reporter stimulated by $100 \mathrm{U} / \mathrm{ml}$ of TNF. d, Expression of Bcl-2 in BAECs abrogated the 40 -fold inducibility of the E-selectin reporter induced by TNF stimulation.

ment of accommodation: rats in one group were splenectomized immediately before transplantation; rats in the second group were not splenectomized. There were 11 rats in the first group, two hearts were removed on days 29 and 37 for study, and two were rejected. The second group included 14 rats, 6 were killed with a beating heart during the first 36 days, and in 3 , the hearts were rejected. Thus, no difference was noted in the two groups in the frequency of either rejection or accommodation.

The next protocols in our study allowed us to determine for how short a period CVF could be given. We treated different groups with CVF for either 9 or 6 days, and again with CyA for the duration of the experiment. Here also we found that the frequencies of rejection or accommodation in the various groups were similar to those found when CVF was administered for 11 days. One of the seven hearts transplanted in these two groups was rejected. We have thus combined the data from all of these groups (treatment with CVF for 6, 9 and 11 days) for the overall discussion of results below.

A total of 33 hamster hearts were transplanted into rats given CyA for the duration of the experiment and CVF daily for 6 to 11 days, starting on day -1 before transplant. Six hearts were rejected on days $5,10,17,21,27$ and 49 (mean $=22 \pm 16$ days). Eight hearts were taken for study while still beating during the first 37 days. The remaining 19 hearts survived for more than 54 days. These hearts were either taken for immunopathology while still beating or are currently surviving. We chose to study the immunopathology of these long-surviving hearts ( $>54$ days) as characterizing accommodation given the high probability that they had accommodated that is, they had survived for longer than the mean of the rejected hearts plus two standard deviations.

\section{Intragraft humoral, cellular and cytokine responses}

Sections of accommodated hearts harvested at 57 days or later posttransplantation showed well preserved myocardial architecture and normal vessels but a moderate-to-dense mononuclear cell infiltrate (Fig. 2). Infiltrating leukocytes, found in large perivascular aggregates and, at a somewhat lower density, diffusely distributed throughout the myocardium, were comparable in distribution, number, composition (mainly macrophages and $\mathrm{CD} 4^{+} \mathrm{T}$ cells) and immune activation (IL-2R expression) in the rejected and accommodated groups (Fig. 2); however, important phenotypic and functional differences were detected (Fig. 2, Table 1). T cells in accommodated grafts lacked CD45RC (OX-22) 


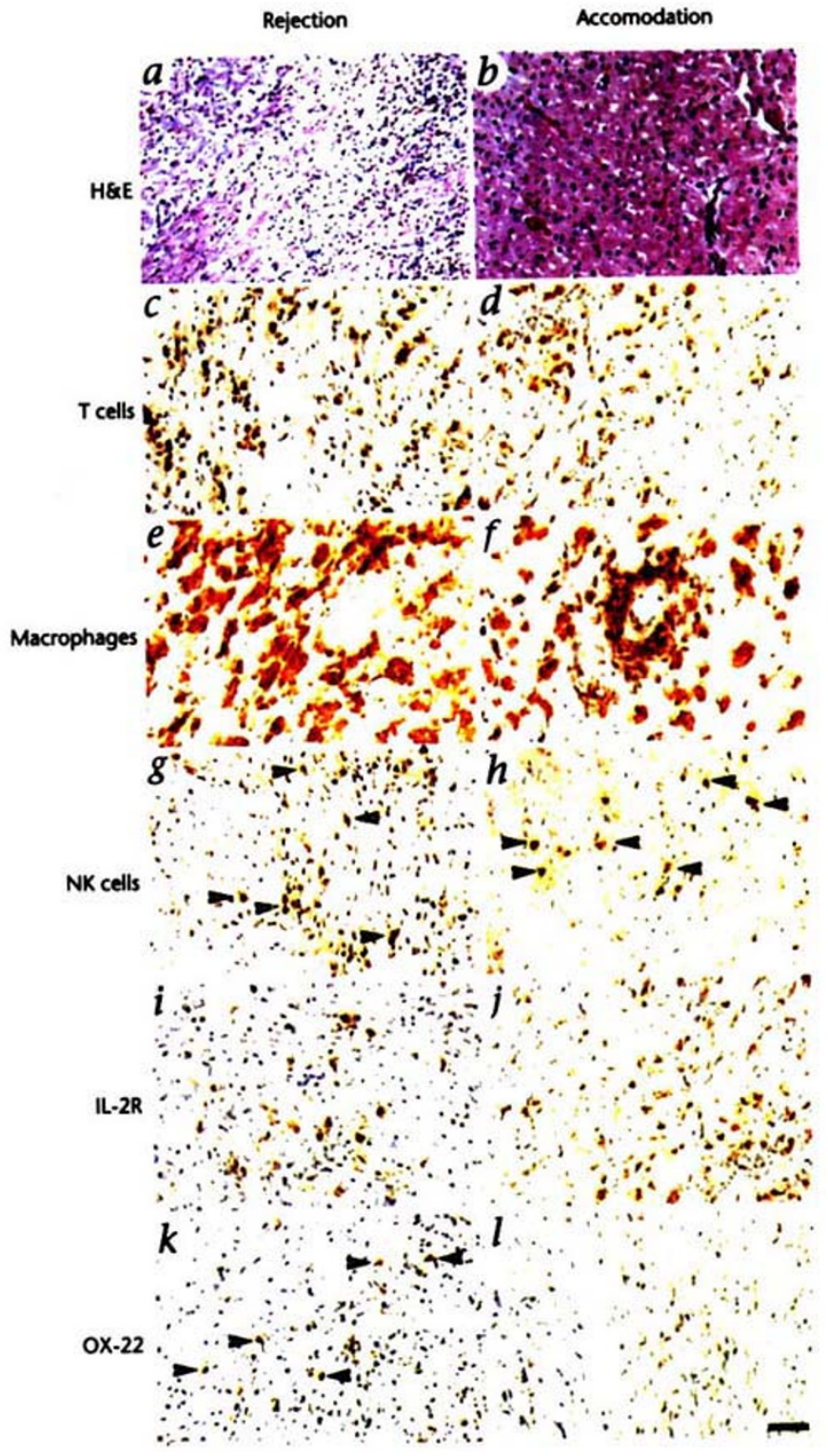

Fig. 2 Histologic features and cells within rejecting versus accommodated xenografts. Hematoxylin and eosin (H\&E)-stained paraffin sections show that (a) rejection is associated with a mononuclear cell (MNC) infiltrate, myocardial fiber disruption and focal necrosis, whereas accommodated grafts $(b)$ also show a MNC infiltrate but otherwise normal histology. Immunoperoxidase staining of cryostat sections shows that both sets of grafts contain comparable numbers of T cells $(c, d)$, inflammatory macrophages $(e, f)$, NK cells (arrows) $(g, h)$, and IL-2 $\mathrm{R}^{+}$leukocytes $(i, j)$, but differ in expression (arrows) of the Th1-associated antigen, OX-22 (CD45RC) ( $k$, D). Scale bar, $100 \mu \mathrm{m}$. These are representative fields from five grafts from each group examined.

expression, a putative marker of Th1 cells $\mathrm{s}^{17-19}$ and, together with associated macrophages and some vascular cells, showed dense expression of IL-4, IL-10 and IL-13 but little or no IL-2, interferon- $\gamma$ (IFN- $\gamma$ ) or TNF- $\alpha$ (Fig. 3). In contrast, intragraft T cells in rejected grafts harvested at 5-49 days posttransplantation expressed CD45RC (Fig. 2), and mononuclear cells showed dense labeling for IL-2, IFN- $\gamma$ and TNF- $\alpha$, but minimal or no IL-4, IL-10 or IL-13 (Fig. 3). Preservation of myocardial structure and function occurred despite the deposition of high titers of IgM, IgG2c, C3 and C5b-9 along the surfaces of graft ECs (Fig. 4, Table 1).

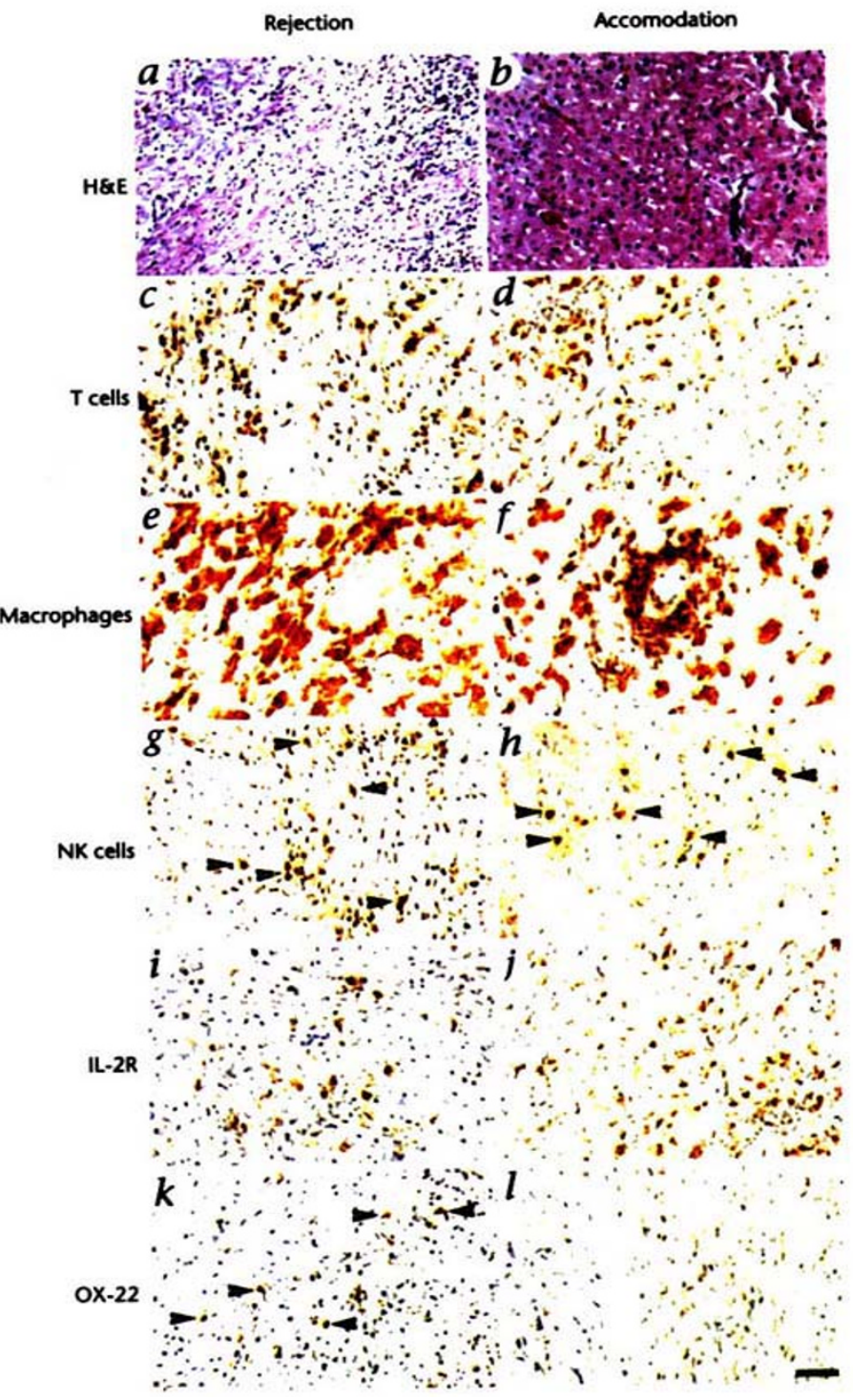

Fig. 3 Cytokine expression within rejecting versus accommodated xenografts. Rejecting but not accommodated grafts show labeling of MNC for IL-2 $(a, b)$, IFN- $\gamma(c, d)$ and TNF- $\alpha(e, f)$; in contrast to rejecting xenografts, accommodated grafts show expression of IL-4 $(g, h)$, IL-10 $(i, j)$, and IL-13 $(k, i)$. Pericellular labeling of leukocytes and some ECs (arrows in panels $c, e, h, j, D$ is also apparent, and residual weak leukocyte labeling in rejecting xenografts for IL-10 (I) and IL-13 $(k)$ is indicated by arrows. (Cryostat sections, hematoxylin counterstain; scale bar, $100 \mu \mathrm{m}$ ). These are representative fields from five grafts from each group examined.

Rejecting xenografts showed similarly high titers of EC-bound IgM, C3, C5b-9, but considerably more IgG1, IgG2a, IgG2b and fibrin, and far less IgG2c, than accommodated grafts (Table 1).

Protective genes and incidence of transplant arteriosclerosis In accommodated grafts, more than $50 \%$ of intragraft large and medium-sized vessels (ECs, SMCs) and $>75 \%$ of the microvasculature showed labeling for $\mathrm{A} 20, \mathrm{Bcl}-2, \mathrm{Bcl}-\mathrm{x}_{\mathrm{L}}$ and hemoxygenase. Myocytes also showed focal expression of hemoxygenase and $b c l-x_{\mathrm{L}}$. These genes were expressed in only rare cells $(<1 \%)$ of rejected 
grafts (Fig. 5), whereas rejected, but not accommodated grafts, showed the pro-apoptotic Bax, Bad and CPP-32 gene products (Table 1). Accommodated grafts also had only basal levels of TUNEL-positive cells, in contrast to the high rates of apoptotic cells that were detected in rejecting xenografts by TUNEL assay (see Methods section, Table 1) and morphologic examination (Fig. 5). Apoptotic cells included small numbers of host leukocytes, as well as SMCs and ECs within intramyocardial branches of the coronary arteries (Fig. 5). Most arteries in rejected grafts showed moderate-to-advanced transplant arteriosclerosis with marked luminal occlusion due to concentrically expanded vascular intimal areas, whereas accommodated grafts lacked evidence of transplant arteriosclerosis (Fig. 5, Table 1).

In summary, accommodated hearts showed a predominance of hemoxygenase, A20, Bcl-2 and the $\mathrm{Bcl}-\mathrm{X}_{\mathrm{L}}$ gene products in their ECs and elsewhere, a Th2 cytokine profile, dense deposition of IgG2c, and a normal vasculature. Rejecting hearts, in contrast, had essentially no expression of the protective gene products but showed the widespread presence of pro-apoptotic genes (Bax, Bad, CPP-32), a Th1 cytokine response, no IgG2c, and a high incidence of transplant arteriosclerosis.

Xenograft resistance to passively transferred rat anti-hamster sera To test the extent to which ECs in the surviving grafts were resistant to the effects of anti-graft antibodies and complement, we challenged rats carrying surviving xenografts by administration of a pooled rat anti-hamster serum that was collected from rats 7 days after transplantation of a hamster heart to an untreated rat, that is, 3 days after rejection. A $0.1-$ or a $0.5-\mathrm{ml}$ dose of this serum resulted in rejection of a hamster heart that had been transplanted into a rat 30 minutes before the injection of the serum: the $0.1-\mathrm{ml}$ dose led to rejection in 240 minutes and the $0.5-\mathrm{ml}$ dose resulted in rejection at 20 minutes. We compared these results with injection of the same serum into rats that had a hamster heart functioning 48 days after transplantation. The $0.1-\mathrm{ml}$ dose did not result in rejection of these hearts; the hearts were removed while still beating on day 62 and studied by immunopathology, which showed a picture not significantly different from the accommodated hearts described in the two paragraphs immediately above. In another rat bearing an accommodated heart for 78 days, $0.1 \mathrm{ml}$ of the same serum was in- jected again without noting rejection over the next 24 hours. However, when an additional $0.4 \mathrm{ml}$ of this anti-hamster serum was injected into the rat, the heart graft was rejected at 7 hours. Both freshly transplanted and accommodated hearts were also rejected when an immune serum taken from rats 21 days after transplantation was injected into the rats, although the accommodated hearts consistently survived longer than the freshly transplanted hearts: for instance, a $0.05-\mathrm{ml}$ dose of this serum resulted in rejection of freshly transplanted hearts in an average of 130 minutes and in rejection of accommodated hearts in an average of 435 minutes.

Second heart grafts transplanted to rats carrying a first transplant To test whether the "environment" in a rat carrying an accommodated hamster heart would influence the survival of a second, freshly transplanted heart, we performed a small series of second transplants to rats carrying a first hamster heart for 24 days or more following transplantation of the first heart; the surviving first heart was left in place. These animals were still receiving CyA but no CVF. Whereas first grafts transplanted to rats receiv- 


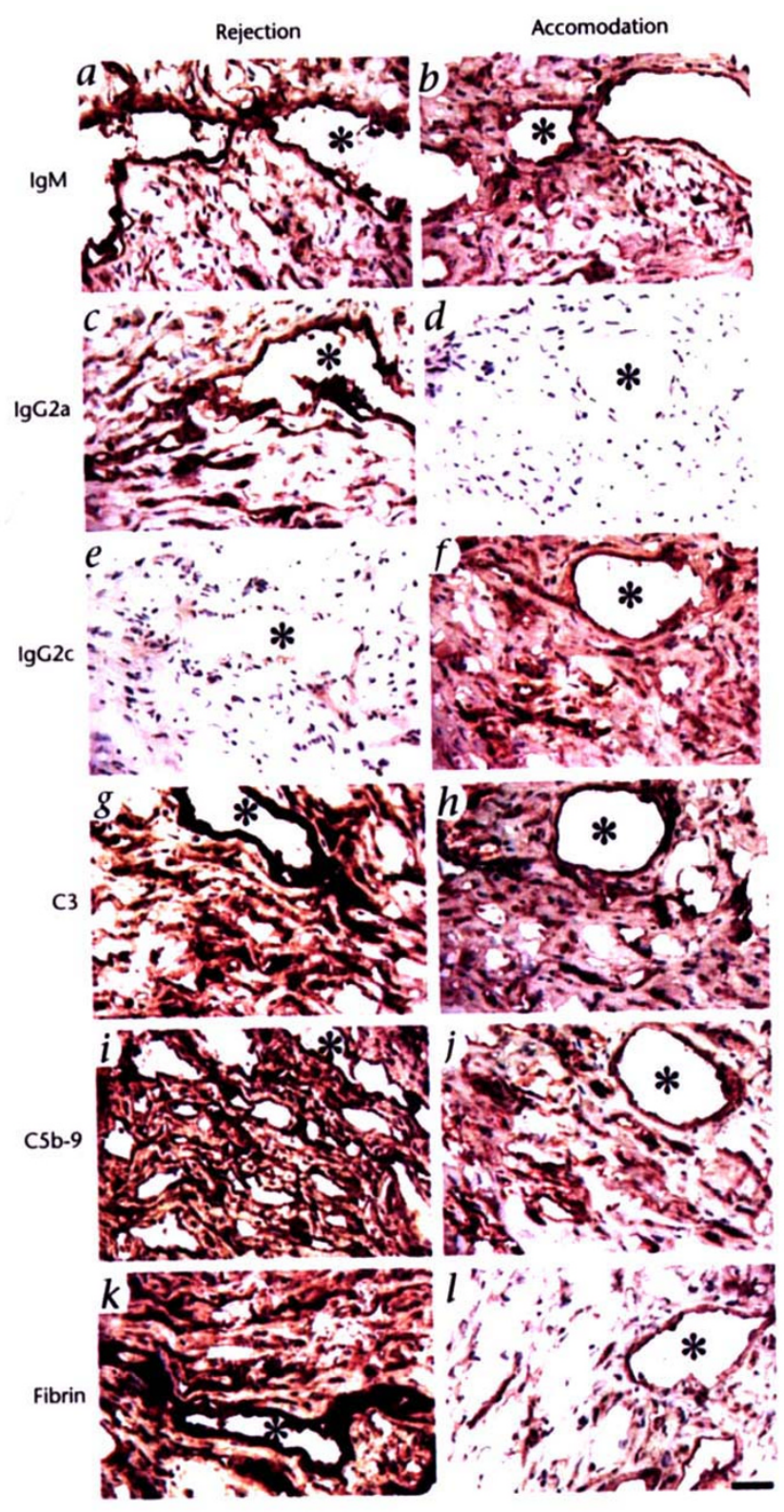

ing only CyA survive for approximately 3 days, all eight second transplants survived 7 or more days. Two of the second transplanted hearts were rejected at 7 and 22 days and showed the immunohistology associated with rejecting of the first hearts. The five long-term surviving second hearts examined had accommodated based on the findings on immunohistology, which were the same as for accommodated first transplants: there was deposition of immunoglobulin and complement on their ECs with a predominant IgG $2 \mathrm{c}$ response, a Th2 cytokine profile and expression of protective genes in their ECs and smooth muscle cells. In two of these five grafts, which were examined at 36 and 39 days posttransplantation, the immunopathology of accommodated hearts already existed, including the expression of the protective genes. One heart was still beating at 52 days.

In order to test whether a first xenograft was required to induce acceptance of a second hamster heart, prospective recipient rats were sham-operated on day 0 and treated for 11 days with CVF and daily CyA until the end of the experiment. When these rats received a cardiac xenograft at day 35 , grafts were rejected at the same pace ( 3 days) as in recipients treated with CyA only. We
Fig. 4 Analysis of EC deposition of immunoglobulins, complement and fibrin within rejecting versus accommodated xenografts, with labeling of the same vessel $\left(^{*}\right)$ in serial sections; the dilution of the primary antibody is indicated in brackets. Both groups have dense IgM labeling $(1: 1000)(a, b)$, whereas the extent of EC labeling for $\lg G 2 a(1: 200)(c, d)$ and $\lg G 2 c(1: 50)$ $(e, f)$ differed markedly. In addition, accommodated grafts showed dense EC deposition of C3 $(1: 1000)(g, h)$, and persistence of EC labeling for C5b$9(1: 500)(i, j)$, but compared with rejecting grafts showed only focal fibrin deposition (1:100) ( $k, 1)$. (Cryostat sections, hematoxylin counterstain; scale bar, $100 \mu \mathrm{m}$ ). These are representative fields from five grafts from each group examined.

hypothesize that it is the Th2 and $\operatorname{lgG} 2 \mathrm{c}$ responses in a rat carrying an accommodated first heart that allow the long-term survival of some of the second hearts.

\section{Discussion}

We have developed a model of concordant xenotransplantation in which heart grafts survive long-term despite the presence of anti-graft antibodies and complement, that is, the grafts accommodate. This model is based on the work of Hasan et al. ${ }^{20}$, who showed in a hamster-to-rat transplantation model that somewhat fewer than $20 \%$ of hamster hearts transplanted into rats treated for 28 days with CVF and daily with CyA for the duration of the experiment survived for 100 or more days despite the presence in the serum of anti-hamster antibodies and complement. These authors reported only survival data, hence, no information is available as to the binding of antibodies and complement to graft ECs.

We varied the length of time for which the recipient rats were treated with CVF to investigate whether we could increase the percentage of hearts that accommodated. We found that treatment of recipient rats with CVF daily for 11 days plus daily and continuing administration of CyA led to accommodation in approximately $75 \%$ of transplants. Splenectomy had no effect on the incidence of accommodation. Even when we treated the rats for only 6 or only 9 days with CVF, and continuously with CyA, accommodation was achieved in more than $75 \%$ of grafts. This model allowed us to examine the intragraft features associated with accommodation: this report is the first description of the expression in ECs and SMCs of "protective genes" associated with graft survival and the avoidance of transplant arteriosclerosis.

Accommodated xenografts displayed excellent morphologic preservation despite the presence of a mononuclear cell infiltrate the extent of which was comparable to the extent associated with rejection of control hearts, and despite the binding of antibodies and complement to graft ECs. Analysis of leukocyte subpopulations present in accommodated and rejected hearts showed that both groups consisted largely of macrophages and somewhat lesser numbers of $\mathrm{CD}^{+} \mathrm{T}$ cells. However, infiltrates in accommodated grafts were markedly skewed toward a Th2 pattern with marked elaboration of the cytokines IL-4, IL-10 and IL-13.

The basis for the immune deviation toward a Th 2 response in these grafts is unclear and not previously noted as a potential factor that might lead to prolongation of xenograft survival. In some rodent cardiac allograft models, CyA therapy can blunt Th1 (IL- 2 and IFN- $\gamma$ production) and leave Th2 responses intact, resulting in long-term survival of grafts $^{21}$. Other immunosuppressive agents, such as rapamycin ${ }^{22}$ can also result in preferential activation of Th2 cells and inhibition of synthesis of complement-binding IgG subclasses, while permitting production of IgG2c in the rat. Alternately, immune deviation can re- 


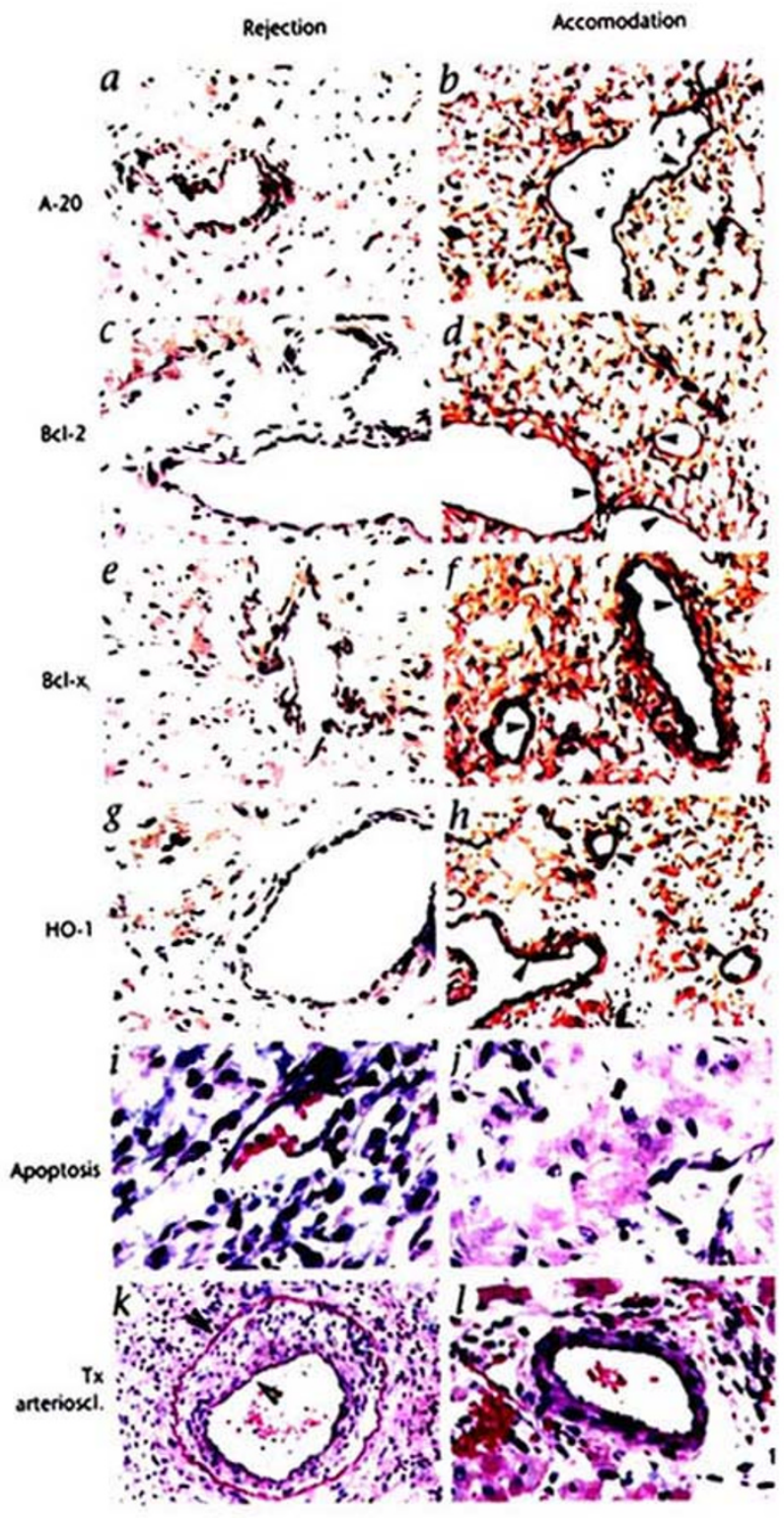

sult from failure of ligation of costimulatory molecules such as $\mathrm{CD} 28$ on responder $\mathrm{CD} 4^{+} \mathrm{T}$ cells by $\mathrm{B} 7$-positive antigen-presenting cells ${ }^{23}$. The Th2 cytokine response in the accommodated hearts likely plays a suppressive response as discussed in several studies of prolongation of allograft survival in the context of an enhanced Th2 cytokine response $\mathrm{e}^{21,2426}$. However, the presence of $\mathrm{Th} 2$ cytokines may have special relevance to the development of accommodation of a xenograft. We have previously noted the presence of monocytes and NK cells, both of which appear to be activated, in rejecting xenografts ${ }^{5,6}$. All three Th2 cytokines (IL-4, IL-10, IL-13) detected in accommodated xenografts suppress the activation of monocytes, though most studies have focused on the potent inhibitory effects of IL-10 (ref. 27); evidence of a similar, albeit less extensively studied, role for Th2 cytokines in regulating NK cell activation is $\mathrm{known}^{28}$. Consistent with these studies are our findings that there are significantly fewer leukocytes expressing TNF- $\alpha$ in the accommodated hearts $(<5 \%)$ than in the rejected hearts $(>50 \%)$, despite similar numbers of macrophages in the two situations.

Our suggestion that an EC protective response might occur
Fig. 5 Protective genes, apoptosis and transplant arteriosclerosis in rejecting versus accommodated xenografts. In contrast to only trace labeling or an absence of labeling in rejecting grafts, accommodated xenografts showed widespread EC labeling (arrows), vascular SMC labeling and in some cases myocyte labeling for A-20 $(a, b), \mathrm{Bcl}-2(c, d), \mathrm{BCl}-\mathrm{x}_{\mathrm{L}}(e, f)$ and hemoxygenase (HO-1) $(g, h)$. In addition, rejecting grafts but not accommodated grafts showed morphologic evidence of apoptosis (arrows) $(i, f)$, and transplant arteriosclerosis (arrows indicate $~ 30 \%$ luminal occlusion due to an expanded intima). Scale bar, $100 \mu \mathrm{m}$. These are representative fields from five grafts from each group examined.

when a graft survived despite the presence of anti-graft antibodies and complement was intended to explain how antibodies and complement could attach to the ECs and yet not evoke the inflammatory response engendered when vascular-based rejection takes place ${ }^{3}$. A protective gene ideally prevents induction of those genes causing inflammation and also protects the ECs from apoptosis. Protecting the ECs of the graft from undergoing apoptosis, which occurs during xenograft rejection (Table 1), is likely exceedingly important. If the ECs die and the subendothelium is exposed, several proteins of that subendothelium will be exposed to the blood elements. Both collagen and von Willebrand factor can combine with glycoprotein gp1b on platelets and thus initiate the process leading to platelet activation and aggregation. The products of activated platelets, including $\mathrm{C}-\mathrm{C}$ chemokines such as MCP-3, will recruit mononuclear cells to the site of rejection; the products of these cells will activate the other systems that appear to cause rejection, including EC activation and thrombosis ${ }^{29}$.

The induction of protective genes in ECs has recently been described in several systems. In terms of preventing induction in ECs of inflammation-causing factors, $\mathrm{we}^{30}$ and others ${ }^{31}$ noted that stimulation of ECs with TNF- $\alpha$, lipopolysaccharide (LPS) or other ligands leads to upregulation of I $\mathrm{KB} \alpha$ in the ECs. I $\mathrm{KB} \alpha$ acts as a natural inhibitor of NF- $\mathrm{KB}$ in resting ECs by binding to the NF- $\mathrm{kB}$ heterodimer and preventing its translocation to the nucleus. As such, the induction and synthesis of I $\mathrm{KB} \alpha$ results in a negative feedback loop to suppress NF- $\mathrm{KB}$, a transcription factor that is critical for the induction of essentially all of the inflammationcausing genes that characterize EC activation ${ }^{13-16}$. With regard to genes that protect the ECs from death, Juckett and colleagues showed that stimulation of ECs with heme leads to induction of ferritin, which protects the ECs from the damage caused by activated neutrophils ${ }^{32}$. Dalmasso and colleagues, while not defining the gene(s) involved, showed that activation of porcine ECs with human xenoreactive natural antibodies, which we have shown can signal the ECs (ref. 33), results in an EC response (dependent on protein synthesis) that protects those cells from lysis by human serum ${ }^{34}$. Most recently, hemoxygenase has been implicated as a inducible protective gene within monocytes and ECs (ref. 35, 36), and indeed, at least two of the four protective genes under consideration (hemoxygenase and $b c l-2$ ) are now recognized to have significant antioxidant properties ${ }^{37-38}$.

Our in vitro data show that the three anti-apoptotic genes studied all inhibit a reporter dependent for its activation only on NF$\kappa \mathrm{B}$ (see the Methods section). Our results from studies in ECs are in accord with other reports in the literature showing that both A20 and Bcl-2, which were studied in other cell types, are able to inhibit the induction of NF-kB activation ${ }^{39,40}$. Using an $A 20$ recombinant adenovirus that is expressed in essentially $100 \%$ of ECs, we were able to confirm the results using reporter assays: expression of $A 20$ in ECs prevented the translocation of NF- $\mathrm{KB}$ to 
the nucleus based on electrophoretic mobility shift analysis (C.F. et al. manuscript in preparation).

To evaluate whether accommodation might be due to the expression of these and other protective proteins in the ECs, we studied expression of the anti-apoptotic gene products: A20, Bcl2 , and Bcl- $\mathrm{X}_{\mathrm{L}}$. More than $50 \%$ of ECs in accommodated hearts expressed $\mathrm{A} 20, \mathrm{Bcl}-2, \mathrm{Bcl}-\mathrm{x}_{\mathrm{L}}$ and hemoxygenase, as did $>50 \%$ of vascular SMCs and 10-20\% of intragraft leukocytes. Organs that were rejected, that is, that did not accommodate, expressed these gene products weakly in only very occasional ECs or SMCs and in $<1 \%$ of leukocytes. Likewise, hamster hearts rejected around day 4 in untreated rats did not express the protective gene products. Consistent with this contrasting pattern of protective gene expression in the accommodated and rejected hearts, the ECs of rejected hearts expressed Bax, Bad and CPP-32, which are associated with apoptosis, whereas ECs in the accommodated hearts did not ( $<1 \%$ of ECs). These data are consistent with the concept that expression in the ECs of the graft of protective genes, in this case the three anti-apoptotic genes just discussed and hemoxygenase, may contribute to achieving accommodation. The reduced fibrin deposition in accommodated versus rejected grafts could reflect lack of tissue factor induction as a result of Th2 cytokine-mediated suppression of macrophage activation, or through an effect in ECs of the NF-kB-inhibiting actions of the anti-apoptotic genes.

The expression of the anti-apoptotic genes may also contribute to the marked difference in the incidence of transplant arteriosclerosis in the two groups under study. Both ECs and SMCs are candidate contributors to the development of arteriosclerosis in many systems, and we found expression of the anti-apoptotic genes in both types of cells within accommodated hearts. In vitro studies show that vascular SMCs display high rates of apoptosis when exposed to proinflammatory cytokines, such as IL- $1 \beta$ or TNF- $\alpha$, or the Th 1 cytokine, IFN- $\gamma$ (ref. 41 ). High rates of apoptosis are noted within coronary arteries of patients with atherosclerosis ${ }^{42}$ and during development of transplant arteriosclerosis in cardiac allograft models ${ }^{43}$. In addition, the inhibition of NF- $\mathrm{KB}$ in SMCs by the products of anti-apoptotic genes (given their similar function in SMCs and ECs) might prevent proliferation of those cells ${ }^{44}$. Prevention of apoptosis and inhibition of NF-kB through the action of anti-apoptotic genes, the expression of which can be favored by a Th2 cytokine environment $\mathrm{t}^{45}$, as reported in vitro, may therefore be of therapeutic significance.

The finding that injection of a rat anti-hamster serum at a dose that causes hyperacute rejection of a freshly transplanted heart in just 240 minutes does not result in rejection of an accommodated heart, unless administered in a quantity several times as great, leads to at least two possible interpretations. The most conservative one is that ECs of the freshly transplanted heart has no antibodies bound to it, and thus, the injected antibodies have immediate and full access to target structures on the ECs. In contrast, the accommodated hearts already have non-complementfixing IgG bound to them, and thus, relatively fewer of the injected antibodies will be able to bind. Given this argument, one could imagine that a dose five times that which causes rejection will result in an increased level of binding of the injected, complement-fixing antibodies, and thus rejection. However, the fact that both rejecting and accommodated hearts already have high levels of IgM, C3 and C5b-9 bound to their ECs suggest to us that hyporesponsiveness (protection) of the graft ECs, while overcome by suprathreshold stimulation by hyperimmune serum, may play a role under the conditions we have noted. Rejection seen consequent to injection of a higher dose of such a serum is probably not due to induction of proinflammatory genes in the ECs, because rejection occurs after just a very few hours. Rather, rejection may involve some of the same phenomena involved in hyperacute rejection of a discordant xenograft. Interactions of subendothelial structures, exposed as the ECs retract from one another, with platelets, and/or loss of EC surface molecules such as heparan sulfate ${ }^{46}$, thrombomodulin ${ }^{47}$, and ATPase $^{48}$ may play a role. In order to avoid these reactions, it may be necessary to inhibit complement.

The demonstration that second hamster heart transplants accommodate in six of the eight cases in rats carrying a first hamster xenograft emphasizes the possibility, if not likelihood, that an ongoing Th2 and/or IgG2c response contributes to the development of accommodation. Second hearts transplanted into rats previously receiving a transplant and treatment with CyA alone did not survive beyond 5 days and show the features on immunohistology of a rejected heart. To the extent that the presence of the IgG2c on the endothelium of the accommodated grafts contributes to, or accounts for, the long-term survival, this would fit the definition of accommodation originally proposed in which the existence of an IgG isotype that fixes complement poorly was envisioned as one possible explanation of accommodation.

Although the causative role of IgG2c, Th2 cytokines and protective genes in allowing the development of accommodation is clearly speculative, the results collectively provide a testable model for factors that may well contribute to the long-term survival of a vascularized (xeno)graft, as well as to the avoidance of transplant arteriosclerosis. For xenotransplantation, in which situation it is possible to create transgenic donor animals, the present findings may find interesting application, as, for instance, by expressing a protective gene(s) in the ECs of the transplant, and being able to regulate the expression of those genes ${ }^{16,49}$. To which extent deviation of the response to a xenograft toward Th2 cells, and attempting to achieve a predominantly IgG2c response (or its equivalent in humans), will contribute to achieving long-term survival of a discordant, immediately vascularized xenograft, will need further investigation.

\section{Methods}

Transfection of bovine aortic endothelial cells (BAECs). BAECs $\left(3 \times 10^{5}\right)$ were plated in $2 \mathrm{ml}$ medium per well in a six-well plate. Cells were transfected once they had reached $70 \%$ confluence. DNA $(1.5 \mu \mathrm{g} /$ well, test plasmids and reporter constructs) was added to $8 \mu \mathrm{g}$ of lipofectamine per well and incubated at room temperature for $30 \mathrm{~min}$ before being added to the cells in triplicate. For all experiments, $0.3 \mu \mathrm{g}$ of the $\beta$-galactosidase ( $\beta$ $\mathrm{gal}$ ) reporter were used, $0.5 \mu \mathrm{g}$ of $\mathrm{A} 20, \mathrm{BCl}-2$ or pAc (the control plasmid), and $0.7 \mu \mathrm{g}$ of the E-selectin and NF-KB reporters, shown in preliminary experiments to be the optimal doses. After a 5 - $h$ incubation, fetal calf serum (FCS) was added to the medium to achieve a final concentration of $10 \%$. Forty-eight hours later the cells were stimulated with either human recombinant TNF- $\alpha(100 \mathrm{U} / \mathrm{ml})$ or LPS $(100 \mathrm{ng} / \mathrm{ml})$ and harvested $7 \mathrm{~h}$ after stimulation. Cellular extracts were assayed for $\beta$-gal and luciferase activity. The E-selectin reporter construct, obtained from C. Brostjan from our laboratories and including base pairs -1286 to +484 of the porcine E-selectin promoter, was cloned into the pMAMneo-luc plasmid vector by replacing the mmTV promoter (Clontech, Palo Alto, CA). The NF-KB reporter was obtained from A. Palmetshofer of our laboratories and consists of four copies of NF-KB elements taken from the porcine E-selectin promoter inserted upstream of a TK minimal promoter driving a luciferase gene. The expression plasmids used were the human $A 20$ and the murine $b c l-2$ genes both cloned in the pAc vector. The $A 20$ gene was obtained from V. Dixit and subcloned into the pAc expression vector. The murine $b c /-2$ gene was obtained from T. Behrens (University of Minnesota) and subcloned in PAC at 
the BamHI site. For calculations, luciferase activity was normalized for $\beta$-gal by using the formula: luciferase activity $/ \beta$-gal activity $\times 1000$. Normalized luciferase activity is given in relative light units (RLU). Significance was determined by the Student's t-test (19).

Animals. Inbred male Lewis rats (225-275 g) and male Golden Syrian hamsters (70-120 g) were purchased from Harlan Sprague-Dawley (Indianapolis, IN). Animals were housed in accordance with guidelines from the American Association for Laboratory Animal Care and research protocols were approved by the Institutional Animal Care and Use Committees of the New England Deaconess and Beth Israel Hospitals.

Transplantation. Surgical procedures were carried out under anesthesia with pentobarbital (30-50 mg/kg i.p.; Abbott, North Chicago, IL). Heterotopic hamster to rat cardiac transplantation was performed by a modification of the microsurgical technique of Ono and Lindsey ${ }^{\text {so }}$. Some recipients were splenectomized immediately before transplantation. Cardiac xenografts were observed visually for the first $30 \mathrm{~min}$ after reperfusion and then monitored daily by abdominal palpation. Rejection was diagnosed by cessation of palpable contractions and confirmed by direct visualization and subsequent histologic examination. After harvest of rejected grafts, animals were killed with an overdose of pentobarbital. In some cases hearts were removed while still beating for study.

Hyperimmune sera. Untreated Lewis rats that had rejected their cardiac hamster xenograft served as donors for rat anti-hamster serum, which was collected on postoperative day 7 and 21 , and decomplemented by heat inactivation $\left(56^{\circ} \mathrm{C}, 30 \mathrm{~min}\right)$. These sera were injected into rats that had either received a fresh hamster heart transplant 30 min earlier or into rats that carried accommodated hearts for varying periods of time $(n=2$ for each dose of each serum tested in freshly transplanted or accommodated hearts).

Drugs. CVF (Quidel, San Diego, CA) was administered at a dose of 60 units $/ \mathrm{kg}$ i.p. on the day before transplantation followed by 20 units $/ \mathrm{kg}$ per day for varying numbers of days as indicated in the text. CyA (Sandoz Pharma, Basel, Switzerland) was given daily ( $15 \mathrm{mg} / \mathrm{kg}$ i.m.) until the end of each experiment.

Antibodies. Monoclonal antibodies (mAbs) obtained from Serotec (Harlan Bioproducts for Science, Indianapolis, IN), except where noted, were directed against all rat leukocytes (CD45, OX-1); $T$ cells ( $T$-cell receptor- $\alpha / \beta, R 73)$; T-cell subsets (CD4, BWH-4, M. Sayegh, Boston, MA; CD8 $\alpha$, OX-8; CD45RC, OX-22); B cells (CD45RA, OX-33); NK cells (NKRP1 , 3.2.3); mononuclear phagocytes (CD68, ED-1); and neutrophils (RP3, F. Sendo, Yamagata, Japan). Cell activation was assessed using mAbs to rat MHC class II antigens (OX-3); IL-2R (CD25, ART-18, T. Diamantstein, Berlin, Germany); IL-1 $\beta$ (SILK-6); IL-2 (1D10), IL-4 (OX81) and IFN- $\gamma$ (DB-12, P. van der Meide, Rijswijk, the Netherlands), plus monospecific polyclonal antibodies to IL-10, IL-13 and TNF- $\alpha$ (R\&D Systems, Minneapolis, MN). Apoptosis-associated gene products were studied using mAbs to Bad, BCl-x and CPP-32 (Transduction Laboratories, Lexington, KY); and Bax (PharMingen, Los Angeles, CA); and rabbit antibodies to $A 20$ (V. Dixit, Ann Arbor, $M N$ ); Bcl-2 (PharMingen); and $\mathrm{BCl}-\mathrm{x}_{L}$ (C. Thompson, Chicago, IL). Rabbit antibodies to rat hemoxygenase-1 (HO-1) and hemoxygenase-2 ( $\mathrm{HO}-2)$ were obtained from StressGen (Victoria, BC, Canada).

Humoral responses were analyzed using mAbs to rat IgA (MARA-2), IgG1 (MARG-2), IgC2a (MARG2a-1), IgG2b (MARG2b-8), IgG2c (MARC2c-2), IgM (MARM-4); C3 (ED-11, Serotec); C5b-9 (W. Couser, Seattle, WA); and a polyclonal antibody to rat fibrin (ICN, Irvine, CA). Developing reagents for immunohistology consisted of rat immunoglobulinabsorbed goat anti-mouse immunoglobulin (Sigma), rabbit anti-goat immunoglobulin, goat peroxidase-anti-peroxidase complex (PAP) for detection of mouse mAbs. Binding of rabbit or goat polyclonal antibodies was detected using swine anti-rabbit immunoglobulin and rabbit PAP, or rabbit anti-goat immunoglobulin and goat PAP, respectively, purchased from Dako (Carpinteria, CA). Details of these mAbs, isotype-matched control mAbs, purified rabbit or goat immunoglobulin controls, and their use in immunohistologic studies of rat recipients of cardiac transplants, were recently described ${ }^{5,2426}$.
Immunopathology and TUNEL. Xenografts were harvested, subdivided and quick-frozen in isopentane and stored at $-80^{\circ} \mathrm{C}$ in preparation for immunohistologic studies, or fixed in neutral-buffered formalin, embedded in paraffin and sectioned for light microscopy. Cryostat sections were fixed in paraformaldehyde-lysine-periodate for demonstration of leukocytes and activation antigens, or fixed in acetone for localization of cytokines, and stained by a three-layer (for polyclonal antibodies) or four-layer (for mAbs) PAP method, as previously described ${ }^{23-25}$. Briefly, sections were incubated overnight with primary antibodies at $4{ }^{\circ} \mathrm{C}$, followed by incubations at room temperature with bridging antibodies, PAP complexes and diaminobenzidine.

Isotype-matched mAbs or purified immunoglobulin, and a control for residual endogenous peroxidase activity, were included in each experiment. Studies were undertaken to confirm the lack of labeling of normal hamster hearts $(n=5)$ with the antibodies used, and positive controls for antibody labeling were provided using sections of rat allografts, confirming that the antibodies were able to detect their specific antigen, the data from which were reported previously ${ }^{23-25}$. Last, key antibody absorption with corresponding recombinant rat cytokines (IL-2, IFN- $\gamma$, IL-4 from Serotec) was undertaken before immunohistologic labeling ${ }^{23-25}$. Apoptotic cells were detected within cryostat sections using a TUNEL kit (Oncor, Gaithersburg, $M D$ ), according to the manufacturer's instructions. Briefly, this technique involves incorporation of biotinylated UTP after incubation with terminal deoxynucleotidyl transferase; nucleotides are detected using peroxidasetagged streptavidin.

Data analysis. A guide to the extent of endothelial deposition of rat $\mathrm{C}$, IgG, IgM and fibrin deposition within hamster xenografts was obtained by comparing the end-titer of labeling obtained following serial dilution of corresponding primary antibodies ${ }^{24,25}$. Numbers of labeled CD45-positive cells and TUNEL-positive cells within 10 high power fields/section per rat $(n=$ 6/group) were determined and compared by the Mann-Whitney U-test. Cytokine and endothelial labeling was judged semiquantitatively, because of the presence of extracellular (cytokines) or continuous (EC) labeling, as $<1 \%, 1-10 \%, 10-20 \%, 20-50 \%,>50 \%$ or $>90 \%$ of the cells indicated, as described $^{23-25}$.

CH-50 assays. Lysis of antibody-sensitized sheep erythrocytes by the classical complement pathway was evaluated by the dilution of rat serum required to produce $50 \%$ maximal cell lysis as measured by the release of hemoglobin.

\section{Acknowledgment}

This work was supported by Sandoz Pharma, Basel, Switzerland and in part by a grant from the National Institutes of Health (A140152).

\section{RECEIVED 26 AUGUST; ACCEPTED 16 DECEMBER 1996}

1. Slapak, M., Digard, N., Ahmed, M., Shell, T. \& Thompson, F. Renal transplantation across the ABO barrier - A 9-year experience. Transplant. Proc. 22, 1425-1428 (1990).

2. Alexandre, G.P.J., Latinne, D., Gianello, P. \& Squifflet, J.P. Preformed cytotoxic antibodies and ABO-incompatible grafts. Clin. Transplant. 5, 583-587 (1991).

3. Bach, F.H. Turman, M.A., Vercellotti, G.M., Platt, J.L. \& Dalmasso, A.P. Accommodation: A working paradigm for progressing towards clinical discordant xenografting. Transplont. Proc. 23, 205-208 (1991).

4. Winkler, H., Ferran, C. \& Bach, F.H. Accommodation of xenografts: A concept revisited. Xenotransplantation 2, 53-56 (1995).

5. Blakely, M.L. et al. Activation of intragraft endothelial and mononuclear cells during discordant xenograft rejection. Transplantation 58, 1059-1066 (1994).

6. Lesnikoski, B.A., Shaffer, D.A., Van der Werf, W.1., Hancock, W.W. \& Bach, F.H. Endothelial and host mononuclear cell activation and cytokine expression during rejection of pig-to-baboon discordant xenografts. Transplant. Proc. 27, 290-291 (1995).

7. Opipari, A.J., Hu, H.M., Yabkowitz, R. \& Dixit, V.M. The A20 zinc finger protein protects cells from TNF cytotoxicity. I. Biol. Chem. 267, 12424-12427 (1992).

8. Tsujimoto, Y. \& Croce, C.M. Analysis of the structure, transcripts and protein products of bcl-2, the gene involved in human follicular lymphoma. Proc. Natl. Acad. Sci. USA 83, 5214-5218 (1986).

9. Boise, L.H. et al. Bcl-x, a bcl-2-related gene that functions as a dominant regulator of apoptotic cell death. Cell 74, 597-608 (1993).

10. Jaattela, M., Mouritzen, H., Elling, F. \& Bastholm, L. A20 zinc finger protein inhibits TNF and IL-1 signaling. J. Immunol. 156, 1166-1173 (1996). 
11. Cooper, J.T. et al. A20 blocks endothelial cell activation through a NF-kappa B-dependent mechanism. J. Biol. Chem. 271, 18068-18073 (1996).

12. Song, H.Y., Rothe, M. \& Goeddel, D.V. The tumor necrosis factor-inducible zinc finger protein A20 interacts with TRAF1/TRAF2 and inhibits NF-kappa B activation. Proc. Natl. Acad. Sci. USA 93, 6721-6725 (1996).

13. Read, M.A., Whitley, M.Z., Williams, A.J. \& Collins, T. NF-кB and $1 \kappa B \alpha-$ An inducible regulatory system in endothelial activation. J. Exp. Med. 179, 503-512 (1994).

14. Ferran, C. et al. Inhibition of NF-kappa B by pyrrolidine dithiocarbamate blocks endothelial cell activation. Biochem. Biophys. Res. Commun. 214, 212-223 (1995).

15. Wrighton, C.J. et al. Inhibition of endothelial cell activation by adenovirus-mediated expression of $I \kappa B \alpha$, an inhibitor of the transcription factor NF-KB. J. Exp. Med. 183, 1013-1022 (1996)

16. Bach, F.H. et al. Barriers to xenotransplantation. Nature Med. 1, 869-873 (1995).

17. Arthur, R.P. \& Mason, D.T. Cells that help B cell responses to soluble antigen are distinguishable from those producing interleukin-2 on mitogenic or allogeneic stimulation. J. Exp. Med. 163, 774-786 (1986).

18. Papp, I. et al. Evidence for functional heterogeneity of rat $\mathrm{CD4}^{+} \mathrm{T}$ cells in vivo Differential expression of IL-2 and IL-4 messenger RNA in recipients of cardiac allografts. I. Immunol. 148, 1308-1314 (1992).

19. Hancock, W.W., Sayegh, M.H., Kwok, C.A., Weiner, H.L. \& Carpenter, C.B. Oral but not intravenous alloantigen prevents accelerated allograft rejection by selective intragraft Th2 cell activation. Transplantation 55, 1112-1118 (1993).

20. Hasan, R., Vandenbogaerde, J.B., Wallwork, J. \& White, D.J.G. Evidence that longterm survival of concordant xenografts is achieved by inhibition of antispecies antibody production. Transplantation 54, 408-413 (1992).

21. Takeuchi, T., Lowry, R.P. \& Konieczny, B. Heart allografts in murine systems - The differential activation of Th2-like effector cells in peripheral tolerance. Transplantation 53, 1281-1294 (1992).

22. Ferraresso, M., Tian, L., Ghobrial, R., Stepkowski, S.M. \& Kahan, B.D. Rapamycin inhibits production of cytotoxic but not noncytotoxic antibodies and preferentially activates Thelper 2 cells that mediate long-term survival of heart allografts in rats. f. Immunol. 153, 3307-3318 (1994).

23. Sayegh, M.H. et al. CD28-B7 blockade after alloantigenic challenge in vivo inhibits Th1 cytokines but spares Th2. J. Exp. Med. 181, 1869-1874 (1995).

24. Hancock, W.W. et al. Blocking of mononuclear cell accumulation, cytokine production and endothelial activation within rat cardiac allografts by CD4 monoclonal antibody therapy. Transplantation 53, 1276-1280 (1992).

25. Kupiec-Weglinski, J.W. et al. CD4 mAb therapy modulates alloantibody production and intracardiac graft deposition in association with selective inhibition of Th1 lymphokines. I. Immunol. 151, 5053-5061 (1993).

26. Mottram P.L., Han, W.R., Purcell, L.J., McKenzie, I.F.C. \& Hancock, W.W. Increased expression of IL-4 and IL- 10 and decreased expression of IL- 2 and IFN- $\gamma$ in long-surviving mouse heart allografts after brief CD4 monoclonal antibody therapy. Transplantation 59, 559-565 (1995).

27. Geng, Y., Gulbins, E., Altman, A. \& Lotz, M. Monocyte deactivation by interleukin 10 via inhibition of tyrosine kinase activity and the ras signaling pathway. Proc. Natl. Acad. Sci. USA 91, 8602-8606 (1994).

28. Naume, B. \& Espevik, T. Review: Immunoregulatory effects of cytokines on natural killer cells. Scand. J. Immunol. 40, 128-134 (1994).

29. Hancock, W.W. Chemokines and the pathogenesis of T cell-dependent immune responses. Am. J. Pathol. 148, 681-684 (1996).

30. de Martin, R. et al. Cytokine-inducible expression in endothelial cells of an $1 \kappa B \alpha-l i k e$ gene is regulated by NF-kB. EMBO /. 12, 2773-2779 (1993).

31. Bellas, R.E., Lee, J.S. \& Sonenshein, G.E. Expression of a constitutive NF-KB-like activity is essential for proliferation of cultured bovine vascular smooth muscle cells. J. Clin. Invest. 96, 2521-2527 (1995).

32. Juckett, M.B. et al. Ferritin protects endothelial cells from oxidized low density lipoprotein in vitro. Am. J. Pathol. 147, 782-789 (1995).

33. Vanhove, B., de Martin, R., Lipp, J. \& Bach, F.H. Human xenoreactive antibodies of the IgM isotype activate pig endothelial cells. Xenotransplantation 1, 17-23 (1994).

34. Dalmasso, A.P., He, T. \& Benson, B.A. Human IgM xenoreactive natural antibodies can induce resistance of porcine endothelial cells to complement-mediated injury. Xenotransplantation 3, 54-62 (1996).

35. Agarwal, A., Kim, Y., Matas, A.J., Alam, J. \& Nath, K.A. Gas-generating systems in acute renal allograft rejection in the rat - Co-induction of heme oxygenase and nitric oxide synthase. Transplantation 61, 93-98 (1996).

36. Willis, D., Moore, A.R., Frederick, R. \& Willoughby, D.A. Heme oxygenase: A novel target for immunomodulation of the inflammatory response. Nature Med. 2, 87-90 (1996).

37. Hockenbery, D.M., Oltvai, Z.N., Yin, X.M., Milliman, C.L. \& Korsmeyer, S.J. Bcl-2 functions in an antioxidant pathway to prevent apoptosis. Cell 75, 241-51 (1993).

38. Vaux, D.L. \& Strasser, A. The molecular biology of apoptosis. Proc. Natl. Acad. Sci. USA 93, 2239-2244 (1996).

39. Jaattela, M., Mouritzen, H., Elling, F. \& Bastholm, L. A20 zinc finger protein inhibits TNF and IL-1 signaling. /. Immunol. 156, 1166-1173 (1996).

40. Grimm, S., Bauer, K.A., Baeuerie, P.A. \& Schulze-Osthoff, K. BCl-2 down-regulates the activity of transcription factor NF-KB induced upon apoptosis. /. Cell Biol. 134, 13-23 (1996)

41. Geng, Y.G., Wu, Q., Muszynski, M., Hansson, G.K. \& Libby, P. Apoptosis of vascular smooth muscle cells induced by in vitro stimulation with IFN- $\gamma$, TNF- $\alpha$, and IL-1 $\beta$. Arterioscler. Thromb. Vasc. Biol. 16, 19-27 (1996).

42. Bennett, M.R., Evan, G.I. \& Schwartz, S.M. Apoptosis of human vascular smooth muscle cells derived from normal vessels and coronary atherosclerotic plaques. $)$. Clin. Invest. 95, 2266-2271 (1995).

43. Shaddy, R. et al. Myocardial apoptosis in a heterotopic murine heart transplant model of chronic rejection and graft vasculopathy. . . Heart Lung Transplant. 15, 597 (1996).

44. Autieri, MV Yue, T. F. Ferstein, G.Z. \& Ohistein, E. Antisense oligonucleotides to the p65 subunit of NFkB inhibit vascular smooth muscle cell adherence and proliferation and prevent neointima formation in rat carotid arteries. Biochem. Biophys. Res. Commun. 213, 827-836 (1995).

45. He, X.W., Zhong, W.Y., Goronzy, J.J. \& Weyand, C.M. Induction of B cell apoptosis by Th0, but not Th2, CD4+ T cells. J. Clin. Invest. 95, 564-571 (1995).

46. Platt, 1.L. et al. Release of heparan sulphate from endothelial cells: Implications for pathogenesis of hyperacute rejection. J. Exp. Med. 171, 1363-1368 (1990)

47. Tsuchida, A., Salem, H., Thomson, N.M. \& Hancock, W.W. Tumor necrosis factor production during human renal allograft rejection is associated with depression of plasma protein $C$ and free protein $\$$ levels and decreased intragraft thrombomodulin expression. J. Exp. Med. 175, 81-90 (1992).

48. Robson, S.C. et al. Potential mechanism of abnormal thromboregulation in xenograft rejection: Loss of ecto-ATPases upon endothelial cell activation. Transplant. Proc. 28, 536 (1996).

49. Gossen, M. \& Bujard, H. Tight control of gene expression in mammalian cells by tetracycline responsive promoters. Proc. Natl. Acad. Sci. USA 89, 5547-5551 (1992).

50. Ono, K. \& Lindsey, E.S. Improved technique of heart transplantation in rats. J. Thorac. Cardiovasc. Surg. 57, 225-229 (1969). 